\title{
Butler Matrix Frequency Diverse Retrodirective Array Beamforming: An Energy-Efficient Technique for mmWave Networks
}

\author{
Shaddrack Yaw Nusenu $(\mathbb{D}$ and Emmanuel Asare \\ Koforidua Technical University (KTU), Koforidua, Ghana \\ Correspondence should be addressed to Shaddrack Yaw Nusenu; nusenu2012gh@yahoo.com
}

Received 20 November 2019; Revised 14 January 2020; Accepted 24 January 2020; Published 22 February 2020

Academic Editor: Enrico M. Vitucci

Copyright (c) 2020 Shaddrack Yaw Nusenu and Emmanuel Asare. This is an open access article distributed under the Creative Commons Attribution License, which permits unrestricted use, distribution, and reproduction in any medium, provided the original work is properly cited.

\begin{abstract}
Millimeter-wave (mmWave) networks with the frequency spectrum ranging from $30 \mathrm{GHz}$ (with wavelength $10 \mathrm{~mm}$ ) to $300 \mathrm{GHz}$ (with wavelength $1 \mathrm{~mm}$ ), can support massive wireless data in fifth-generation (5G) systems. Importantly, large colocated antenna elements can be exploited at the base station (BS) to facilitate beam-steering synthesis along the users' directions. This paper proposes the Butler matrix (BM) frequency diverse retrodirective array (BM-FDRA) beamforming network at the BS for multiuser communications in mmWave networks. We utilize the orthogonal feature of the $M \times M$ BM with $M$ elements of the FDRA to create directional beams for concurrent transmission towards different users in range-angle locations. The proposed scheme has the following merits: (a) there is beam-steering orthogonality without beam interferences and (b) there is automatic tracking functionality, i.e., the prior knowledge on the user location is not required by the proposed BM-FDRA at the BS. The proposed method can serve multiple users concurrently using the beam-steering orthogonality property. Furthermore, performance metrics such as the multiaccess secrecy sum rate (SSR) model, bit error rate (BER), system capacity, and energy efficiency are examined. The proposed BM-FDRA scheme achievements are highlighted via simulation results.
\end{abstract}

\section{Introduction}

The recent growth in mobile devices as well as the use of Internet mobile applications has called for higher data capacity. In particular, mmWave networks offered an attractive approach to meet this challenge due to their highfrequency spectrum from $30 \mathrm{GHz}$ (with wavelength $10 \mathrm{~mm}$ ) to $300 \mathrm{GHz}$ (with wavelength $1 \mathrm{~mm}$ ) [1-5]. In the fifth generation $(5 \mathrm{G})$, the employment of the large-frequency spectrum (i.e., mmWave bands) can be a realistic solution for communications [6-11]. However, since the mmWave has high attenuation (i.e., path loss), an antenna array with high directivity beam gain should be used to overcome the attenuation and absorption. Additionally, steerable and configurable antennas are required to support communications at different user locations [12]. Fortunately, because of small wavelength in the mmWave, the base station (BS) can accommodate large elements in a closely limited space [6]. Consequently, a suitable beamforming technique can be designed to enhance the energy efficiency and transmission range in mmWave networks [13].

In mmWave networks, antenna design technologies, for example, multiple-input multiple-output (MIMO) and/or massive MIMO, and spatial division multiple access (SDMA) can be realized $[6,14]$. In [15], the authors considered beamforming and SDMA techniques for downlink communications (i.e., IEEE 802.11ac).

The existing mmWave communications in the literature focused on point-to-point application scenarios, for instance, wireless personal area network (WPAN) [16], cellular backhaul [17], or wireless local area network (WLAN) [18]. Also, another technique, called beamspace MIMO, has been 
investigated widely by several authors [19-22]. Very recently, the study [10] proposed the multiuser transmission technique in $\mathrm{mmW}$ ave communications.

Up to date, researches on mmWave communication applications adopted phased-array antennas (PAs). Though the PA can provide directional beam gain, only angular information directions are available for all the ranges (i.e., it has range-independent energy focusing). Note that mmWave propagation channels are usually correlated [23]. For instance, if the channels of two receiving users are highly correlated (i.e., colocated), using the PA, the signalto-noise ratio (SNR) of the users may be degraded. Consequently, in certain mmWave practical applications, the ability to control users' information along the angle-rangedependent dimension is extremely useful, especially in mmWave directional beam-steering, suppression of range interference, and user tracking and/or detection. Hence, it is necessary to consider new antenna array beam-steering for mmWave communications.

Therefore, we propose the Butler matrix (BM) frequency diverse retrodirective array (BM-FDRA). BM $[24,25]$ is a Fourier transform technique which consists of $M$ input ports and $M$ output ports with passive four-port power couplers and fixed phase shifters. Note that the BM is another form of beamforming network which can be utilized to excite $M$ array elements to steer the beams towards any of $M$ spatial directions. The BM has been widely applied in communication applications $[26,27]$. Another new beam array, namely, FDA proposed in [28], has a small frequency increment across each element to provide angular-range-time-dependent focusing [29]. In contrast, the PA lacks range-time-focusing transmit beam-steering. Actually, the FDA has been widely investigated in the radar fields [30-38]. Recently, because of FDA potentials, it has been utilized in communication applications $[27,39-43]$. Thus, using the FDA, we achieve angular-rangetime beam dependence. In addition, range-dependent interference or noise suppression is feasible.

Moreover, we can improve the beam-steering-focusing ability by employing the retrodirective array (RDA). This is because the RDA has the merit of automatically returning a signal back towards the direction from where it originated. The RDA technology was overviewed in [44]. In the literature, the RDA has been adopted in several applications [45-51]. And this paper has taken the advantage of RDA features to mmWave communication applications for the first time.

Therefore, motivated by the BM beam orthogonality feature, FDA angular-range-dependent focusing transmit beamforming, and RDA self-steering arrays (i.e., the prior knowledge on the user locations is not required for the BS), we propose an energy-efficient orthogonal beamforming network for concurrent transmissions towards different users using the Butler matrix $(\mathrm{BM})$ frequency diverse retrodirective array (BM-FDRA) at the BS for mmWave communications. In summary, we present the main novelties as follows:

(1) We propose an energy-efficient directional orthogonal two-dimensional (2D) (i.e., angular-range dimension) beamforming network based on the Butler matrix (BM) frequency diverse retrodirective array (BM-FDRA) in mmWave communications. Note that the prior knowledge on the user locations is not required by the BS. Therefore, we can achieve better energy efficiency at the BS. The beamforming transmission is done concurrently without mutual interferences due to the orthogonality features of the generated beams. Therefore, we can serve multiple users within short range in mmWave communications, as depicted in Figure 1.

(2) The secrecy sum rate (SSR) and bit error rate (BER) are analysed for the mmWave communications. Other performance metrics, such as system capacity and energy efficiency, have been examined. We make corresponding comparisons with the PA and other techniques in the literature to validate the achievements of the proposed BM-FDRA scheme in mmWave communications.

\section{Brief Introduction of the Frequency Diverse Array (FDA)}

2.1. FDA Signal Analysis. The FDA beamforming is a nascent array arrangement which has more degrees of freedom than PA beamforming. In the FDA, a small linear/nonlinear frequency increment $\Delta f(t)$ is added across each element. Hence, we can formulate the signal radiated from each element as [29]

$$
s_{m}(t)=w_{m} \exp \left(-j 2 \pi\left(f_{0}+\Delta f_{m}(t)\right) t\right),
$$

where $w_{m}$ denotes the transmit weight and $f_{m}(t)=f_{0}+$ $\Delta f_{m}(t)$ is the radiated frequency, with $m=0,1,2, \ldots$, $M-1$. The carrier frequency is denoted as $f_{0}$, while a nonlinear frequency increment is denoted as $\Delta f_{m}(t)$. In order to generate beamforming towards the desired user location, a propagation time delay constraint expressed as $t \in\left[(r / c), T_{d}+(r / c)\right]$ is required [32], with $T_{d}$ being the pulse duration. Note that when $\Delta f_{m}(t)=m \Delta f(t)$, a linear frequency increment is obtained. The array factor (AF) of the standard FDA is determined as [29-32]

$$
\begin{aligned}
\operatorname{AF}(\theta, r, t)= & \sum_{m=0}^{M-1} \exp \left(\frac{j 2 \pi m d \sin \theta}{\lambda}\right) \times \exp \left(j 2 \pi \Delta f_{m}\left(\frac{t-r_{m}}{c}\right)\right) \\
& \cdot\left(t-\left(\frac{r-m d \sin \theta}{c}\right)\right),
\end{aligned}
$$

where $c, r, r_{m} \approx r-m d \sin \theta$, and $d$ are the light speed, range (i.e., distance), approximated range for the $m$ th element, and element spacing, respectively. More importantly, when $\Delta f(t)=0,(2)$ can be reduced to the array factor (AF) of the PA determined as

$$
\operatorname{AF}(\theta, 0,0)=\sum_{m=0}^{M-1} \exp \left(\frac{j 2 \pi m d \sin \theta}{\lambda}\right) .
$$




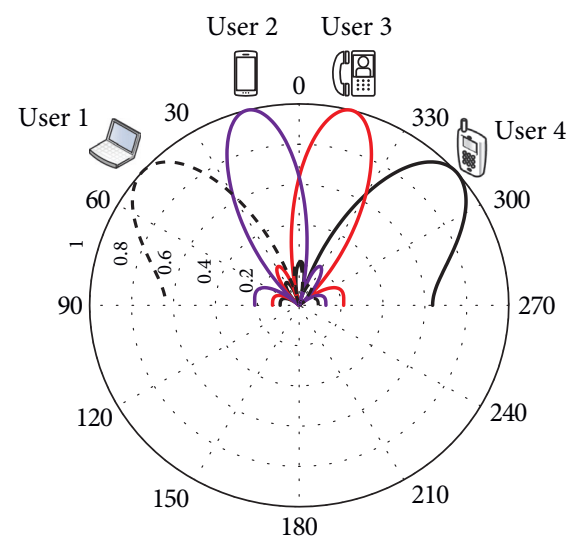

(a)

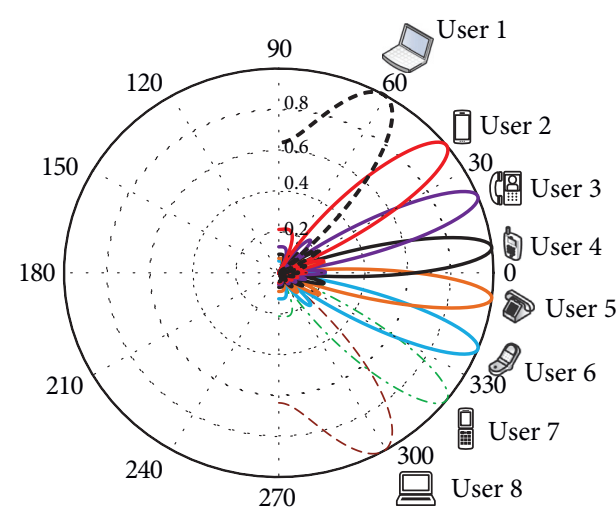

(b)

FIGURE 1: Schematic illustration of the proposed BM-FDRA in mmWave communications: (a) $4 \times 4$ BM-FDRA with orthogonal beams for serving four users concurrently; (b) $8 \times 8$ BM-FDRA with orthogonal beams for serving eight users concurrently. Note the proposed BMFDRA attempts to transmit information using the orthogonal beams over the same frequency band simultaneously to different users. This system model can be extended to any $M \times M$.

Using (2) and (3), respectively, we plot both FDA and PA beamforming in Figure 2. Interestingly, we can see in Figure 2(a) that FDA beamforming is very attractive than PA beamforming depicted in Figure 2(b). This is due to the fact that, with the FDA, we achieve decoupling beamforming at a prespecified user position, while in the PA, we achieve a beamforming with single angle information for all the ranges. Hence, FDA beam-steering is more useful in short-range mmWave communications. This is the motivation of this paper.

\subsection{Brief Introduction of the Retrodirective Array (RDA).} Before deriving the proposed BM-FDRA signal formulations in mmWave systems in Section 3, we present briefly the RDA principle. The capability of the RDA is that it can retransmit a signal back in the direction(s) along which it was illuminated [48]. This implies that the prior knowledge on the user location(s) is not required by the $\mathrm{BS}$ (transmitter). In the RDA, the phase conjugator (PC) is the core element which is used to aid automatic tracking. Herein, we use an example (see Figure 3) to explain briefly the basic principle of the RDA. We consider two users (i.e., user 1 and user 2) located at different positions. These users emit pilot signals, namely, $s_{1}(t)$ and $s_{2}(t)$, at $f_{0}$ (i.e., incident frequency). At the RDA side ( $m^{\text {th }}$ element), the detected signal is determined as $s_{1}(t) h_{1}(t)+s_{2}(t) h_{2}(t)$, where $h_{1}(t)$ and $h_{2}(t)$ are the channel propagation for user 1 and user 2 , respectively. After processing the detected signal via a PC system, we have $\left[s_{1}(t) h_{1}(t)\right]^{*}+\left[s_{2}(t) h_{2}(t)\right]^{*}$. Now, reradiating $\left[s_{1}(t) h_{1}(t)\right]^{*}+\left[s_{2}(t) h_{2}(t)\right]^{*}$ is weighted by the local signal $\Psi(t)$ at the RDA with the frequency $f_{i}$. Hence, the received signal at user 1 is described as

$$
\left[y_{1}(t)\right]_{f_{i}}=\sum_{m=0}^{M-1} \Psi(t)\left\{\left[s_{1}(t) h_{1}(t)\right]^{*}+\left[s_{2}(t) h_{2}(t)\right]^{*}\right\} h_{1}(t)
$$

where ${ }^{*}$ denotes the conjugate operation. When $f_{i}=f_{0}$, with the assumption of channel reciprocity, (4) can be reformulated as

$$
\left[y_{1}(t)\right]_{f_{i}=f_{0}}=\Psi(t) s_{1}^{*}(t) \sum_{m=0}^{M-1}\left|h_{1}(t)\right|^{2}+\Psi(t) s_{2}^{*}(t) \sum_{m=0}^{M-1} h_{2}^{*}(t) h_{1}(t)
$$

Similarly, the received signal at user 2 can be derived and is omitted here for brevity. Note that, in case $f_{i} \neq f_{0}$, the study [49] pointed out that we can compensate for their frequency differences by directly linking them to make (5) still hold for the proposed scheme.

Herein, we highlight the achievements of the proposed BM-FDRA for mmWave networks which is distinct from the existing methods in the literature:

(1) There is no need for user(s) estimation/detection algorithm including CSI because of the adopted RDA in the proposed scheme. Furthermore, there is automatic tracking functionality, i.e., the prior knowledge on the user locations is not required by the BS (transmitter).

(2) Millimeter wave channels are correlated and not independent and identically distributed (i.i.d.) Rayleigh [23]. Hence, it is possible for the user channel to be highly correlated. But in the proposed BM-FDRA, due to the decoupling feature, such correlation is resolved.

(3) The proposed method can serve multiple users concurrently using the beam-steering orthogonality property because of the Butler matrix (BM) beamforming network.

\section{Proposed Butler Matrix (BM) Frequency Diverse Retrodirective Array (BM-FDRA) System Model}

Figure 4 illustrates the proposed BM-FDRA block diagram, while Figure 5 shows the proposed $4 \times 4$ BM-FDRA transmitter architecture at the BS. We employ four FDRA 


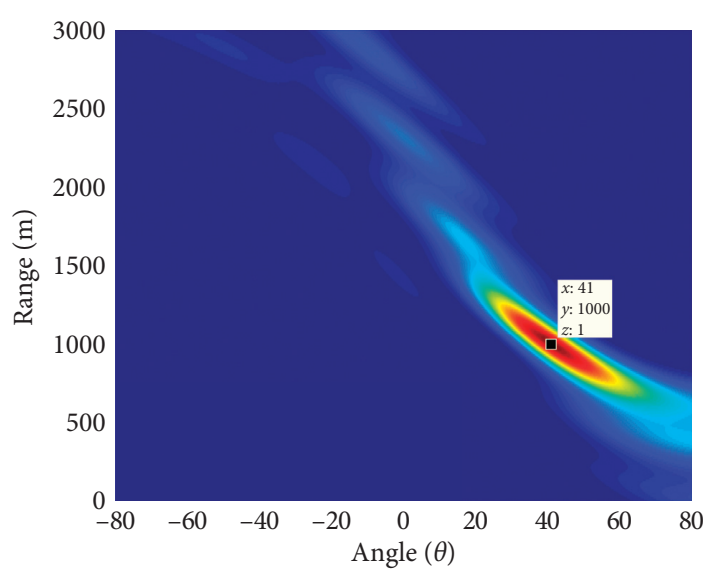

(a)

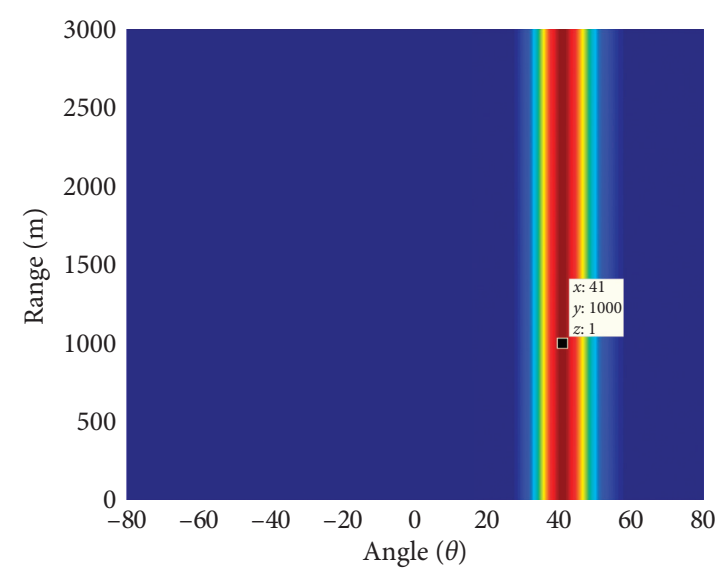

(b)

Figure 2: (a) FDA beamforming along $(\theta, r)=\left(41^{\circ}, 1000 \mathrm{~m}\right)$; we adopted the log-frequency increment to support a few hundred meters in mmWave systems. (b) PA beamforming (i.e., only angle-focusing dependence with the same frequency $f_{0}$ across the element index). Parameters: $M=16, f_{0}=30 \mathrm{GHz}$, and $\Delta f=30 \mathrm{kHz}$.

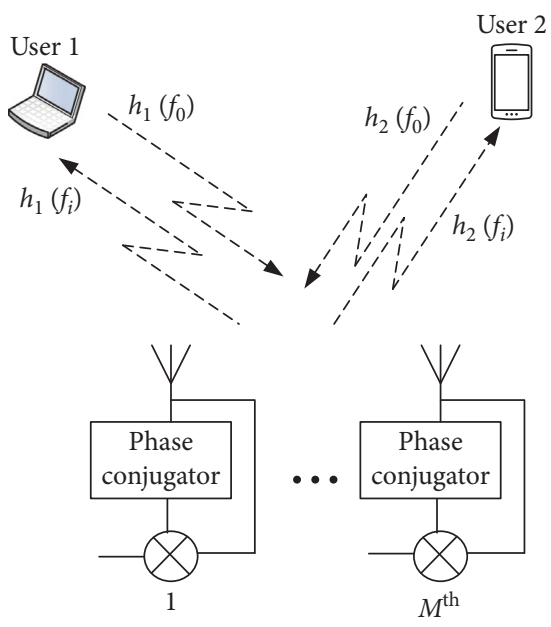

FIGURE 3: Illustration of the RDA principle.

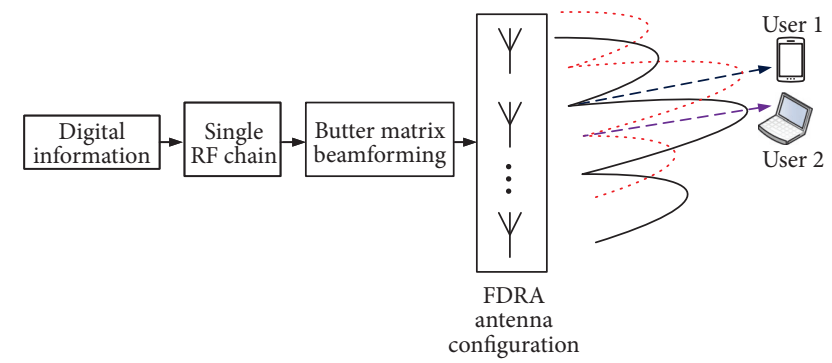

Figure 4: Proposed BM-FDRA block system design.

array elements connected at the $4 \times 4$ Butler matrix (note that it can be extended to any $M \times M$ ) output with carrier frequencies, namely, $2 f_{0}+\Delta f_{0}(t), 2 f_{0}+\Delta f_{1}(t), 2 f_{0}+$ $\Delta f_{2}(t)$, and $2 f_{0}+\Delta f_{3}(t)$, respectively. The operation is briefly described as follows: When Port 1 gets excited, the signal path (i.e., A to $\mathrm{B}$ to $\mathrm{C}$ to $\mathrm{D})$ goes to $2 f_{0}+\Delta f_{0}(t)$ and produces $135^{\circ}$ phase shift. Likewise, we can have $90^{\circ}$ phase shift between Port 1 and $2 f_{0}+\Delta f_{1}(t)$ for the signal path (i.e., A to B to C to E) and so on. For an ideal $4 \times 4 \mathrm{BM}$, output port 1 and 4 phase differences and output port 2 and 3 phase differences are $\pm 45^{\circ}$ and $\pm 135^{\circ}$, respectively [52].

In Figure 6, we have shown the antenna configuration of the FDRA connected at the output of the BM. Note that the principle is described in Section 2.2. Importantly, we exploit both the FDA and the RDA to achieve unique properties in mmWave communications. Furthermore, time slots are designed at the BS to facilitate information beamforming along the respective user locations (see Figure 7).

To proceed with the proposed scheme derivations, we make the following standard assumptions in this paper:

(1) The user(s) radiates pilot signaling to the BS.

(2) There is perfect synchronization between the proposed BM-FDRA transmitter at the BS and the users.

(3) Due to the short transmission distance in mmWave communications, we focus on transmitting orthogonal beams to multiple users in range-angle-focusing directions. It should be noted that we ignore the transmission distance effects in this present work.

\section{2-Dimensional (Range-Angle-Focusing) Orthogonal Beamforming for mmWave Networks}

In this paper, we assume a multiuser communication in mmWave networks. The proposed BM-FDRA transmitter at the BS is equipped with an $M$-element antenna that transmits orthogonal beams concurrently with information to the respective users. Herein, the users are equipped with a single receive antenna, as illustrated in Figures 5 and 7 .

The designed time frame structure depicted in Figure 7 has two transmission phases. Phase $\mathrm{I}$ is allocated time duration $T_{\tau}$, and phase II is also allocated time duration $1-T_{\tau}$. During phase $\mathrm{I}$, the user(s) transmits 


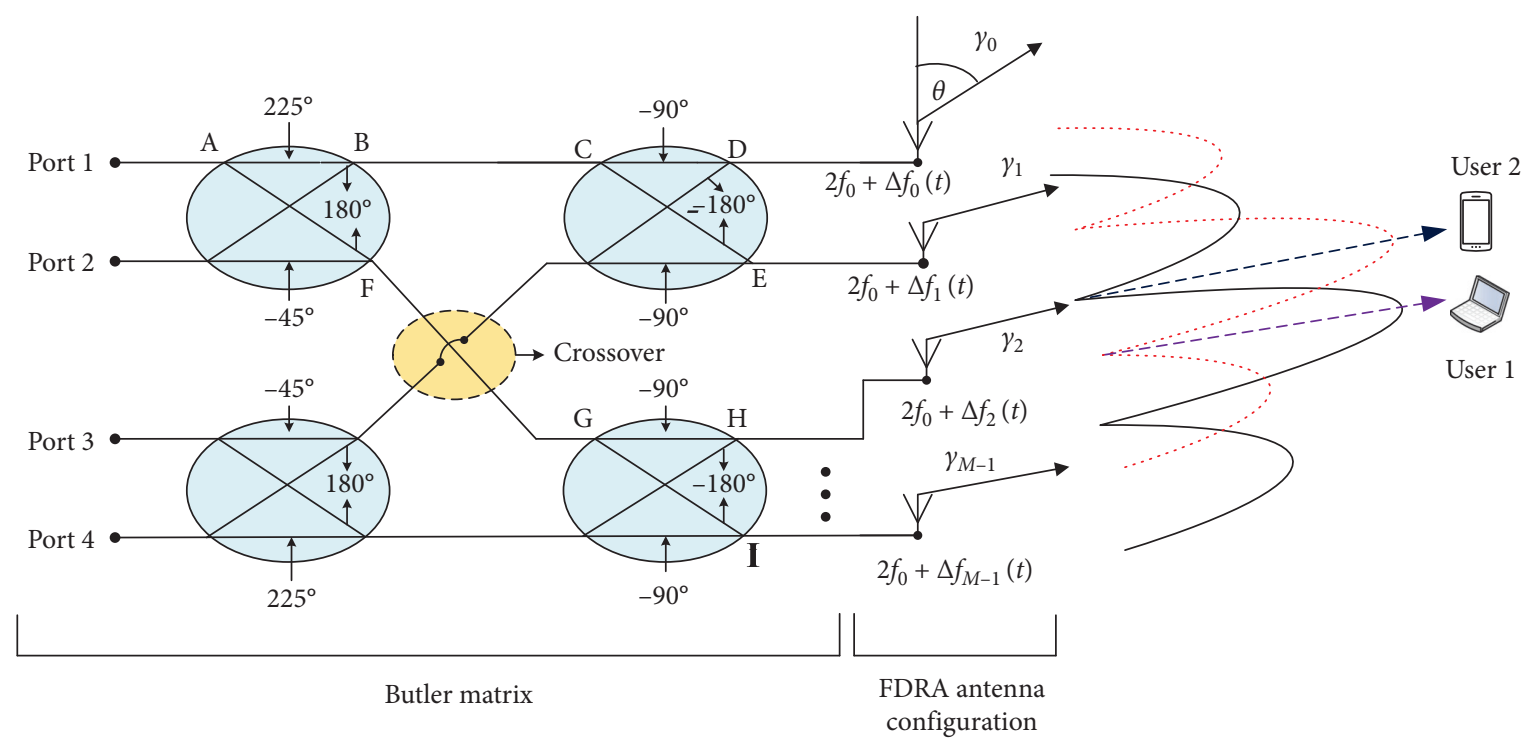

FIGURE 5: Architecture of the proposed $4 \times 4$ BM-FDRA system.

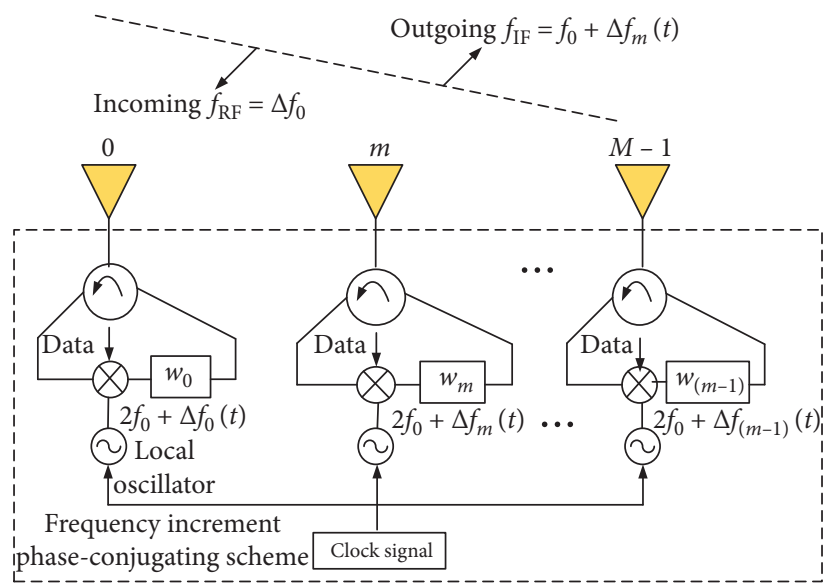

FIGURE 6: Illustration of the FDRA antenna scheme connected at the output of the Butler matrix (BM) system.

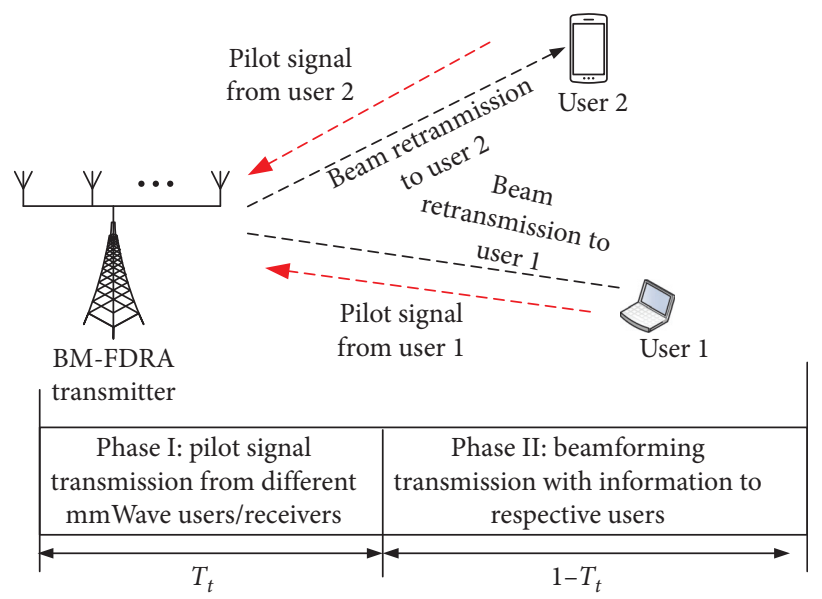

Figure 7: Proposed BM-FDRA time frame structure at the BS (phase I, i.e., $T_{\tau}$ for user pilot transmissions to the BM-FDRA transmitter, and phase II, i.e., $1-T_{\tau}$ BM-FDRA transmitter transmits orthogonal beams to the users). 
pilot signals to the BS. This enables downlink channel estimations. In contrast, in phase II, the BS retransmits the signals with modulated communication data using directional orthogonal beams concurrently to the respective users within the short-range-angle dimension in mmWave networks.

4.1. BM-FDRA Range-Angle Focusing. During phase I, we assume that the user(s) emits phase of the $k$ th $(k=$ $0,1,2, \ldots, K-1)$ pilot signal which is received by the proposed BM-FDRA at the BS with the $m$ th $(m=0,1, \ldots, M-1)$ array element at a particular time $t$. The received pilot signals can be determined as

$$
\Psi_{k, m}(t)=2 \pi f_{0}\left(t-\frac{r_{k, m}}{c}\right)
$$

where $r_{k, m}=\tilde{r}-m d \sin \theta_{i n}^{k}$, with $\tilde{r}$ being the range between the $k$ th user and the proposed BM-FDRA first reference element. $\theta_{i n}^{k}$ (i.e., $k$ th pilot signal's incoming angle) originated from the $k$ th user. The proposed BM-FDRA $m$ th array detects the signals which mix with the local oscillator (LO) signal $f_{\mathrm{LO}}(t)=2 f_{0}+\Delta f_{m}(t)$, where $\Delta f_{m}(t)$ denotes the frequency increment (i.e., $\Delta f_{m} \ll f_{0}$ ).
In phase II, the retransmitted signals by the proposed BM-FDRA at the BS can be written as

$$
\begin{aligned}
y_{n}(t ; R, \theta)= & \sum_{k=0}^{K-1} w_{m} \cdot a(t)\left(T_{m n} \cdot B_{n}\right) \exp j 2 \pi\left[\left(f_{0}+\Delta f_{m}\right)\right. \\
& \left.\cdot\left(t-\frac{R_{k, m}}{c}\right)+f_{0} \frac{r_{k, m}}{c}\right]
\end{aligned}
$$

where $B_{n}$ is the $n$th Butler matrix input port excitation, $w_{m}$ is the phase weighting, as shown in Figure $6, a(t)$ is the communication information, and $T_{m n}$ is defined as $[26,27]$

$$
\begin{array}{r}
T_{m n}=\frac{1}{\sqrt{M}} \cdot \exp \left(-j \frac{2 \pi}{M}\left(m-\frac{M+1}{2}\right)\left(n-\frac{M+1}{2}\right)\right), \\
m, n=0,1, \ldots, M-1 .
\end{array}
$$

Note that the noise term was not considered. And $R_{k, m}=$ $R-m d \sin \theta$ has the same physical meaning as in (2). The main beam arriving at each user location can be determined in (9), and we assume that $a(t) \approx a\left(t-\left(R_{k, m} / c\right)\right)$ is a slow signal.

$$
\begin{aligned}
y_{n}^{k}\left(t ; R, \theta=\theta_{\text {in }}^{k}\right)= & a\left(t-\frac{R_{k, m}}{c}\right) \exp \left(j 2 \pi f_{0}\left(t-\frac{R-\tilde{r}}{c}\right)\right) \sum_{k=0}^{K-1} \sum_{m=0}^{M-1} w_{m}\left(T_{m n} \cdot B_{n}\right) \exp j 2 \pi\left[\Delta f_{m}\left(t-\frac{R_{k, m}}{c}\right)\right. \\
& \left.\cdot\left(t-\frac{((R-\tilde{r})-m d \sin \theta)}{c}\right)+f_{0} m d \frac{\sin \theta-\sin \theta_{\text {in }}^{k}}{c}\right] .
\end{aligned}
$$

It is important to mention that the expressions in (7) and (9), respectively, are based on the $n$th input port excitation Butler matrix. Hence, the main beam is directed towards $\left(R_{n}, \theta_{n}\right)$ directions.
Similarly, by replacing $n$ in (7) and (9), respectively, with $q$, we can also derive expressions for other user locations based on the $q$ th Butler matrix input port excitation with the main beam pointing towards $\left(R_{q}, \theta_{q}\right)$ directions as

$$
\begin{aligned}
y_{q}^{k}\left(t ; R, \theta=\theta_{\text {in }}^{k}\right)= & a\left(t-\frac{R_{k, m}}{c}\right) \exp \left(j 2 \pi f_{0}\left(t-\frac{R-\widetilde{r}}{c}\right)\right) \sum_{k=0}^{K-1} \sum_{m=0}^{M-1} w_{m}\left(T_{m q} \cdot B_{q}\right) \exp j 2 \pi\left[\Delta f_{m}\left(t-\frac{R_{k, m}}{c}\right)\right. \\
& \left.\cdot\left(t-\frac{((R-\tilde{r})-m d \sin \theta)}{c}\right)+f_{0} m d \frac{\left.\sin \theta-\sin \theta_{\text {in }}^{k}\right] .}{c}\right]
\end{aligned}
$$

It is easy to verify that $y_{n}^{k}\left(t ; R_{q}, \theta_{q}=\theta_{\text {in }}^{k}\right)=0$ when $n \neq q$. This implies that the $y_{q}^{k}\left(t ; R, \theta=\theta_{\text {in }}^{k}\right)$ main beam is projected along the null radiation direction of $y_{n}^{k}\left(t ; R, \theta=\theta_{\text {in }}^{k}\right)$. Using (9) and (10), we can achieve orthogonal range-angle beamforming to realize multiuser transmissions in mmWave networks. This implies that the $y_{n}^{k}\left(t ; R, \theta=\theta_{\text {in }}^{k}\right)$ main beam is pointing along user 1 located at a different position, while the $y_{q}^{k}\left(t ; R, \theta=\theta_{\text {in }}^{k}\right)$ main beam is pointing 
along the null direction towards user 2 concurrently. Note that the issue of mutual interference is alleviated in the proposed BM-FDRA due to the orthogonality property of beamforming.

\section{Theoretical Performance Evaluation}

5.1. Secrecy Rate Analysis. To evaluate the proposed BMFDRA scheme secrecy performance, we employ the secrecy sum rate (SSR) [53]. The proposed BM-FDRA SSR can be determined as

$$
C_{s}=\sum_{k=0}^{K-1}\left[C_{k}\left(\theta_{k}, R_{k}\right)-\max _{p} C_{P}\left(\theta_{e_{p}}, R_{e_{p}}\right)\right]^{+} .
$$

The operator $[x]^{+}$returns zero if $x$ is negative or else $x$ is returned. And the secrecy rate from the proposed BM-FDRA to the $k$ th desired user can be computed as

$$
\begin{aligned}
C_{k}\left(\theta_{d_{k}}, R_{d_{k}}\right) & \triangleq I\left(y\left(\theta_{d_{k}}, R_{d_{k}}\right) ;\left[a_{k},\left(\theta_{d_{k}}, R_{d_{k}}\right)\right]\right) \\
& =\log _{2}\left[1+\gamma\left(\theta_{d_{k}}, R_{d_{k}}\right)\right],
\end{aligned}
$$

where $a_{k}$ is the receiving useful information. The instantaneous secrecy capacity at the $k$ th user is given as $\gamma\left(\theta_{d_{k}}\right.$, $\left.R_{d_{k}}\right)=\sigma_{d_{k}}^{-2}\left|\mathbf{w}^{H} \mathbf{a}\left(\theta_{d_{k}}, R_{d_{k}}\right)\right|^{2}$.

Similarly, the secrecy rate from the proposed BM-FDRA to the $p$ th eavesdropper $e_{p}$ is determined as

$$
\begin{aligned}
C_{p}\left(\theta_{e_{p}}, R_{e_{p}}\right) & \triangleq I\left(y\left(\theta_{e_{p}}, R_{e_{p}}\right) ;\left[a_{k},\left(\theta_{e_{p}}, R_{e_{p}}\right)\right]\right) \\
& =\log _{2}\left[1+\gamma\left(\theta_{e_{p}}, R_{e_{p}}\right)\right] .
\end{aligned}
$$

The instantaneous secrecy capacity at the pth eavesdropper is $\gamma\left(\theta_{e_{p}}, R_{e_{p}}\right)=\sigma_{e_{p}}^{-2}\left|\mathbf{w}^{H} \mathbf{a}\left(\theta_{e_{p}}, R_{e_{p}}\right)\right|^{2}$. Note that $\sigma_{d_{k}}^{-2}$ and $\sigma_{e_{p}}^{-2}$ are the noise powers at desired users and eavesdroppers, respectively.

5.2. Bit Error Rate (BER) Analysis. To evaluate the BER of the proposed BM-FDRA scheme, we adopt the quadrature phase-shift keying (QPSK) modulation format. According to the study in [54], we can compute the symbol error probability of QPSK as

$$
P_{\mathrm{QPSK}}=\operatorname{erfc}\left(\sqrt{\frac{\left(E_{s} / N_{0}\right)}{2}}\right)-4^{-1} \cdot \operatorname{erfc}^{2}\left(\sqrt{\frac{\left(E_{s} / N_{0}\right)}{2}}\right),
$$

where erfc is the complementary error function, determined as $\operatorname{erfc}(x)=(2 / \sqrt{\pi}) \int_{x}^{\infty} \exp (-t)^{2} \mathrm{~d} t$. Since the proposed BM-FDRA utilizes beamforming information-based transmission, the channel gain will be the square of the absolute array far-field beampattern [55]. Hence, we can write the average symbol error probability as

$$
\begin{aligned}
P_{e}(\theta, R, t)= & \mathbb{E}\left\{\operatorname{erfc}\left(\sqrt{\frac{\left(E_{s} / N_{0}\right)\left|\mathrm{AF}_{\mathrm{BM}-\mathrm{FDRA}}(\theta, R, t)\right|^{2}}{2}}\right)\right. \\
& \left.-\frac{1}{4} \operatorname{erfc}^{2}\left(\sqrt{\frac{\left(E_{s} / N_{0}\right)\left|\mathrm{AF}_{\mathrm{BM}-\mathrm{FDRA}}(\theta, R, t)\right|^{2}}{2}}\right)\right\},
\end{aligned}
$$

where $\mathbb{E}$ is the expectation operator taken with respect to the number of beams.

5.3. Capacity Evaluation. The primary objective of wireless networks is to provide massive connectivity of users with a higher information rate. It is well known that, by improving the signal-to-interference-plus-noise-ratio (SINR), the information rate can be enhanced. The proposed BM-FDRA scheme has the capability to improve the SINR as well as the capacity of the mmWave communication link. The capacity can be expressed as

$$
C_{y}=\log _{2}(1+\sin R) \text {. }
$$

Since the proposed BM-FDRA scheme depends on angle, range, and time dimensions, we can derive the SINR in three different ways as follows:

$$
\begin{aligned}
& \operatorname{SINR}_{\text {BM-FDRA }}=\frac{\Phi_{s}^{2}\left|\mathbf{w}^{H} \mathbf{a}(r, t)\right|}{\Phi_{n}^{2} \mathbf{w}^{H} \mathbf{w}}, \\
& \operatorname{SINR}_{\text {BM-FDRA }}=\frac{\Phi_{s}^{2}\left|\mathbf{w}^{H} \mathbf{a}\left(\theta=\theta_{\text {in }}, t\right)\right|}{\Phi_{n}^{2} \mathbf{w}^{H} \mathbf{w}}, \\
& \operatorname{SINR}_{\text {BM-FDRA }}=\frac{\Phi_{s}^{2}\left|\mathbf{w}^{H} \mathbf{a}\left(\theta=\theta_{\text {in }}, t\right)\right|}{\Phi_{n}^{2} \mathbf{w}^{H} \mathbf{w}} .
\end{aligned}
$$

For the comparison purpose, the PA SINR is given as

$$
\operatorname{SINR}_{\mathrm{PA}}=\frac{\Phi_{s}^{2}\left|\mathbf{w}^{H} \mathbf{a}\left(\theta=\theta_{\text {in }}\right)\right|}{\Phi_{n}^{2} \mathbf{w}^{H} \mathbf{w}},
$$

where SNR $=\Phi_{s}^{2} / \Phi_{n}^{2}$, with $\Phi_{s}^{2}$ being the signal power and $\Phi_{n}^{2}$ being the noise power. It is important to mention that the main difference between (17)-(19) and (20) is that the PA method provides the angle-based SINR only, while the proposed BM-FDRA scheme provides the angle-rangetime-based SINR. Furthermore, interferences/noise can be suppressed better in the proposed BM-FDRA scheme than in the PA method, thus improving the capacity.

\section{Simulation and Discussion}

In this section, we present several simulations to highlight the potentials of the proposed BM-FDRA scheme in mmWave communication networks. 


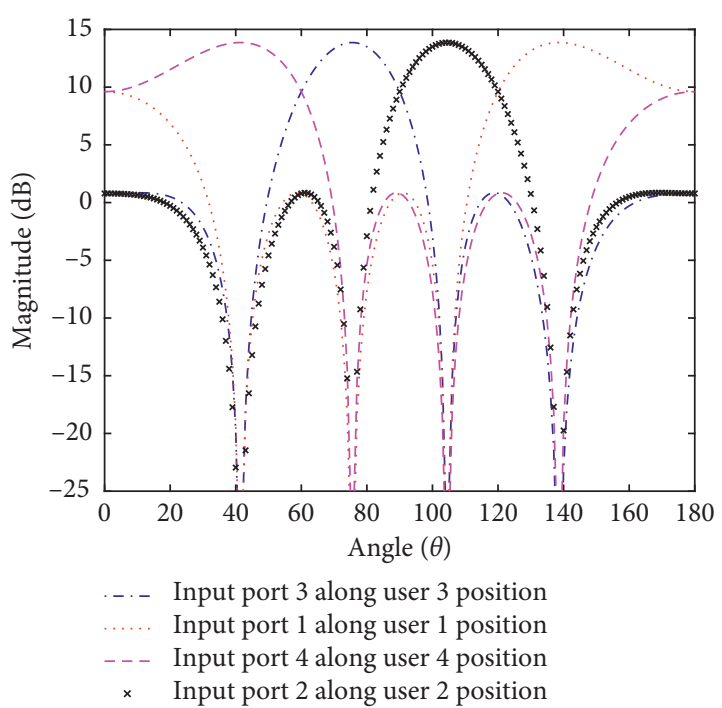

(a)

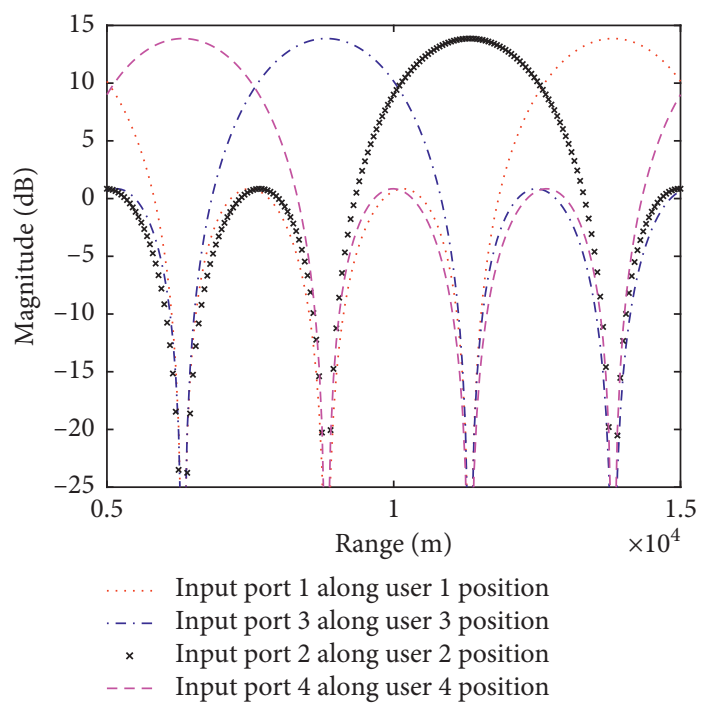

(b)

FIGURE 8: Illustration of the proposed $4 \times 4$ BM-FDRA beamforming network along $k$ users in the (a) angle profile and (b) range profile. Parameters: $M=4, f_{0}=30 \mathrm{GHz}$, and $\Delta f=20 \mathrm{kHz}$.

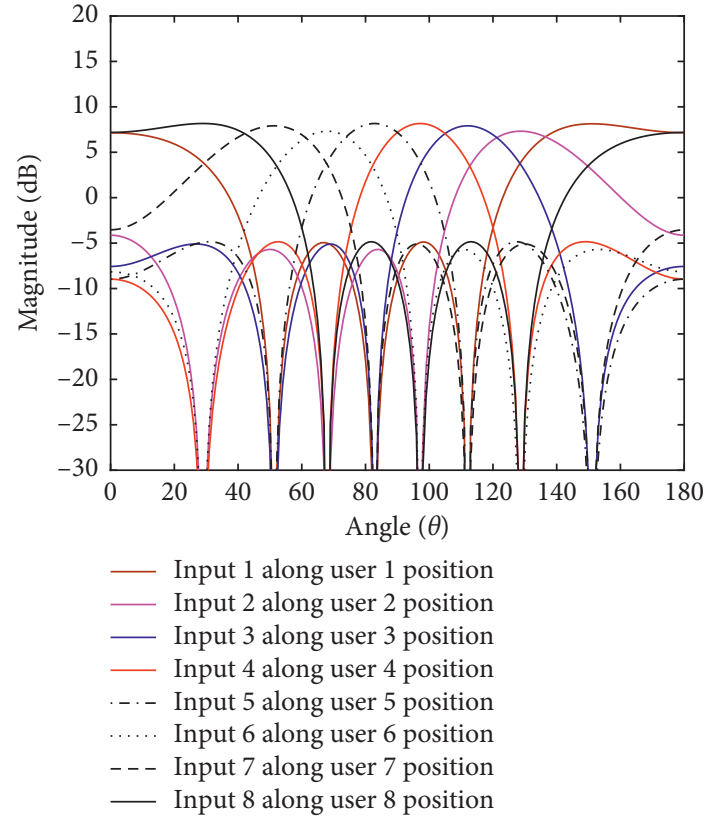

(a)

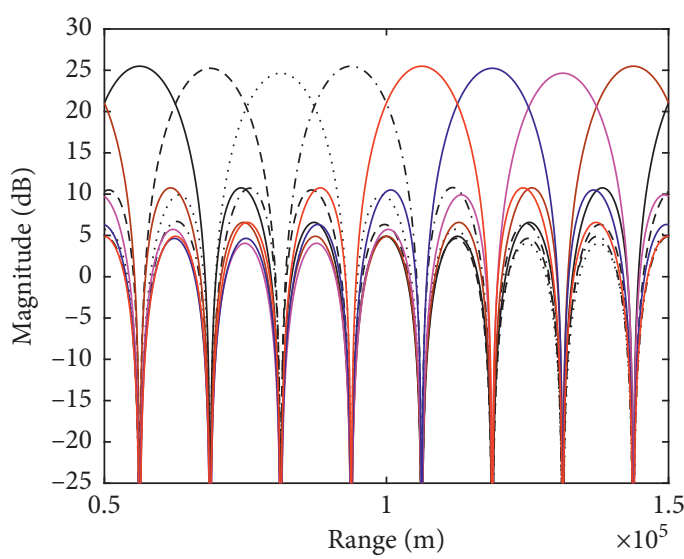

...- Input 5 along user 5 position Input 6 along user 6 position

- - - Input 7 along user 7 position

_ Input 8 along user 8 position

— Input 1 along user 1 position

— Input 2 along user 2 position

- Input 3 along user 3 position

_ Input 4 along user 4 position

(b)

FIgURE 9: Illustration of the $8 \times 8$ beam-steering towards $k$ users in the (a) angle profile and (b) range profile. Parameters: $M=8$, $f_{0}=30 \mathrm{GHz}$, and $\Delta f=20 \mathrm{kHz}$.

(a) The proposed $4 \times 4$ BM-FDRA beamforming network towards $\mathrm{k}$ users at distinct locations in mmWave communications: in Figures 8(a) and 8(b), we have shown the proposed BM-FDRA beamforming networks in angle and range profiles, respectively. Here, four users can be considered with four input port excitations, "1," " 2 ," “3," and " 4 ," respectively. We can see that, in the angle dimension, as depicted in Figure $8(\mathrm{a})$, we achieve orthogonal beams along four distinct angle directions, namely, $41^{\circ}, 76^{\circ}, 104^{\circ}$, and $139^{\circ}$. Similarly, in Figure 8(b), orthogonal beams along different range values are generated, namely, $6.2 \mathrm{~km}, 8.9 \mathrm{~km}, 11.2 \mathrm{~km}$, and $13.9 \mathrm{~km}$. Note that based on Figure 8, we can assign users to each directional beam direction.

(b) The proposed $8 \times 8$ BM-FDRA beamforming network towards $\mathrm{k}$ users at distinct locations in mmWave communications: in Figure 9, we can 


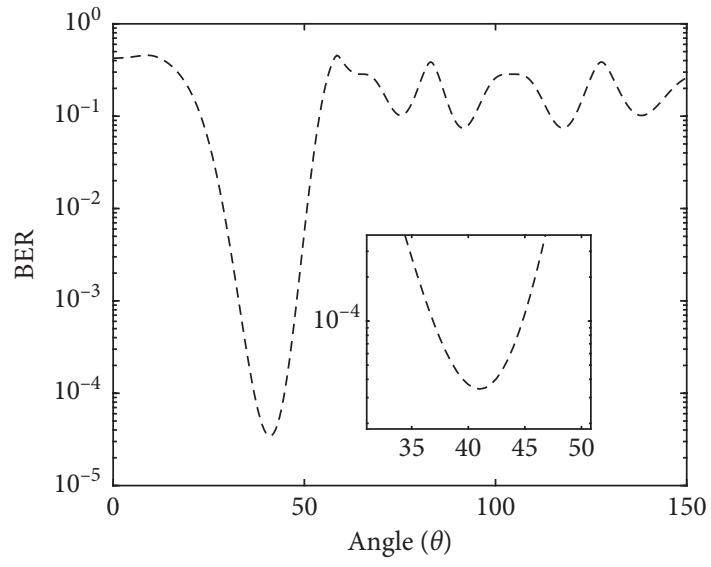

(a)

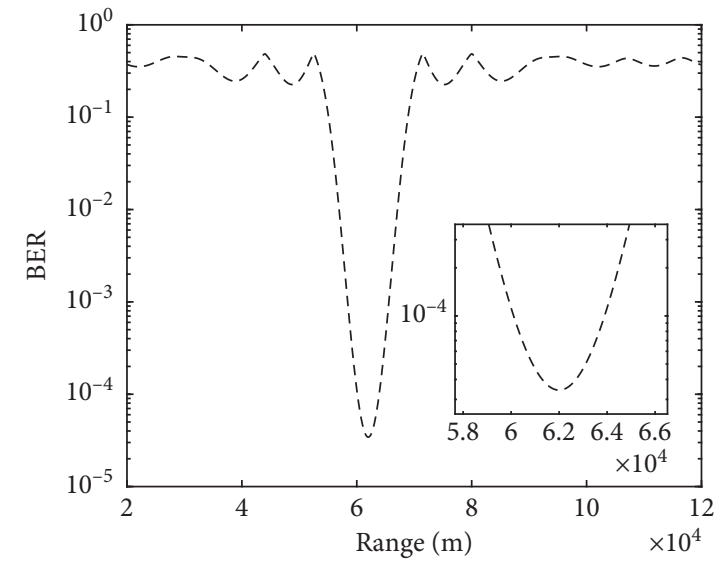

(b)

Figure 10: BER distribution beamforming in the (a) angle profile and (b) range profile. Parameters: user location $\left(41^{\circ}, 6.2 \mathrm{~km}\right), \mathrm{SNR}=6 \mathrm{~dB}$, $f_{0}=30 \mathrm{GHz}$, and $\Delta f=10 \mathrm{kHz}$.

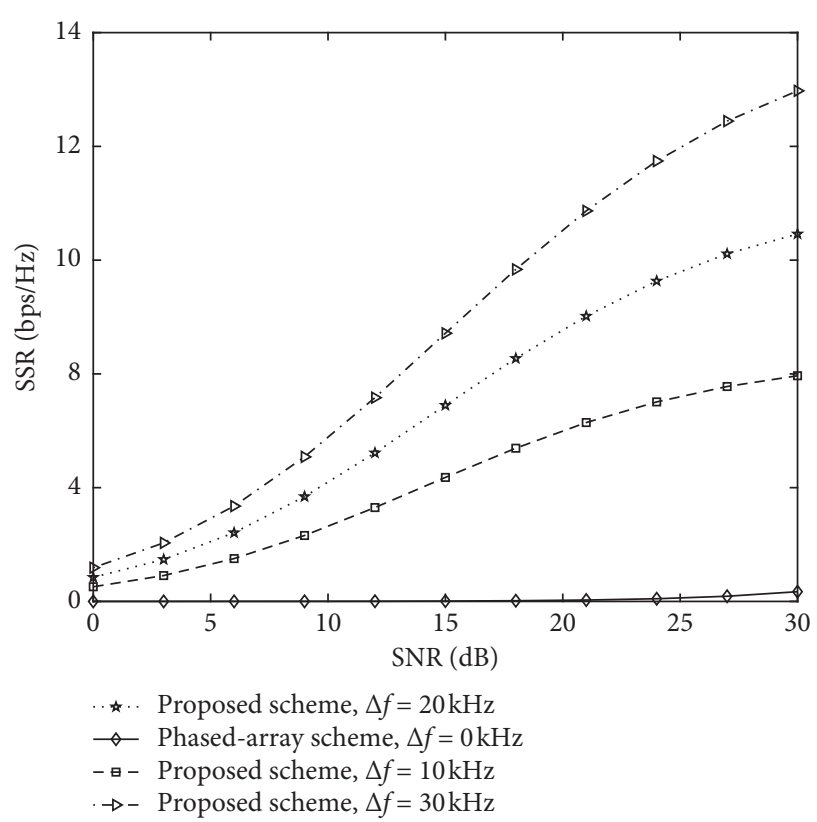

FIGURE 11: SSR as a function of SNR with different frequency increment values: $\Delta f=0$ (PA), $\Delta f=10, \Delta f=20$, and $\Delta f=30$, for the desired user $\left(41^{\circ}, 6.2 \mathrm{~km}\right)$ and eavesdropper $\left(41^{\circ}, 7.5 \mathrm{~km}\right)$.

employ 8 users using the proposed BM-FDRA in mmWave networks. Note that Figures 9(a) and 9(b) give a similar trend, as shown in Figure 8. It is important to remark that the proposed BM-FDRA scheme can be extended to any $M \times M$ orthogonal beam-steering.

(c) BER beamforming along a particular user: suppose one of the users in the mmWave network is located at $\left(41^{\circ}, 6.2 \mathrm{~km}\right)$. Figure 10 demonstrates the BER beamforming along such a location in angle and range dimensions. We observe that Figure 10(a) (i.e., angle dimension) has narrow beamwidth along $41^{\circ}$ with relatively low sidelobes which can minimize

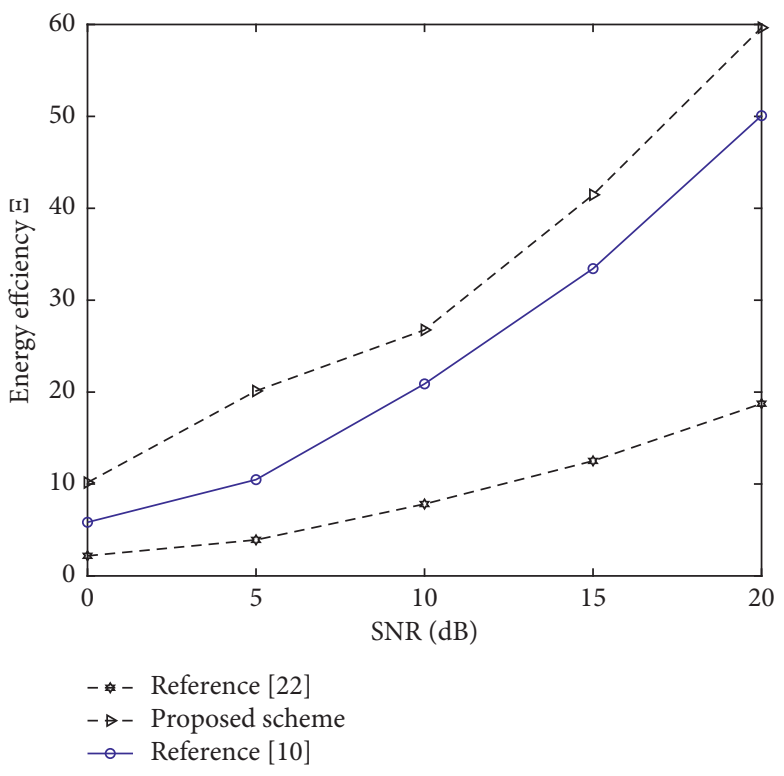

FIGURE 12: Energy efficiency versus SNR. We assume 8 users.

interference with other users. Similarly, in the range dimension along $6.2 \mathrm{~km}$ (see Figure 10(b)), we notice a similar trend.

(d) The proposed BM-FDRA SSR analysis: Figure 11 presents the curves of the SSR against the SNR assuming the desired user and eavesdropper are located in the same direction but distinct ranges. From the figure, the PA SSR curve is zero. This is because the PA is range-independent, hence achieving poor SSR. In contrast, the proposed BM-FDRA scheme with different frequency increment values, namely, $\Delta f=10, \Delta f=20$, and $\Delta f=30$, respectively, has better SSR curves than the PA curve. This is due to the fact that the proposed BM-FDRA has the ability to discriminate between two users along angle-range focusing. Additionally, it can be seen that increasing 


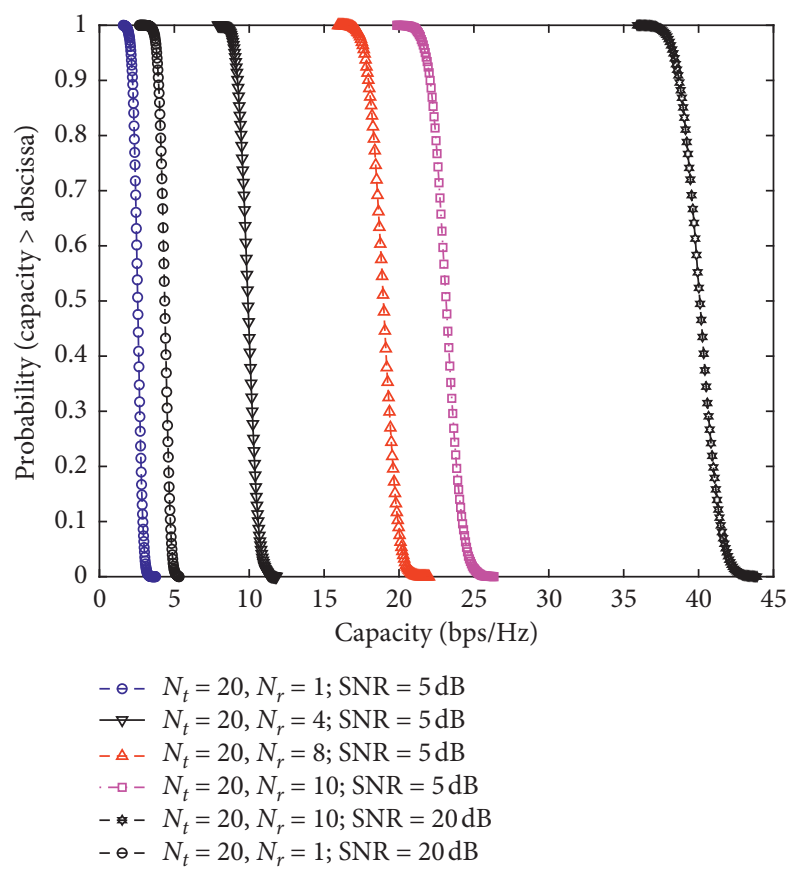

FIgURE 13: Cumulative density function (CDF) curves of capacity for different SNR values: $5 \mathrm{~dB}$ and $20 \mathrm{~dB}$.

TABLE 1: Summary of characteristics of the proposed BM-FDRA scheme and methods in $[10,22]$.

\begin{tabular}{lccc}
\hline Characteristics & Proposed BM-FDRA & {$[10]$} & Improved \\
\hline Energy efficiency & Very improved & No & Good \\
Automatic self-tracking & Yes & Angle only & Angle only \\
Range-angle beamforming & Both & Assumed & No \\
User location & Precise location & Yes & No \\
Gain flexibility adjustment & Yes & No & No \\
Beam orthogonality property & Good & No \\
Range-dependent interference suppression & & & No \\
\hline
\end{tabular}

the values of frequency increments has better influence on the SSR curves. Thus, SSR performance can be enhanced significantly.

(e) Energy efficiency analysis: finally, the proposed BMFDRA energy efficiency is evaluated. According to the study in [56], we can define the energy efficiency $\Xi$ as

$$
\Xi=\frac{C_{s}}{P_{\text {total }}},
$$

where $C_{s}$ is defined in (11) and $P_{\text {total }}=P_{t}+$ $M_{\mathrm{RF}} P_{\mathrm{RF}}+P_{\text {baseband, with }} P_{t}$ denoting the total transmitted power, $P_{\mathrm{RF}}$ denoting the power consumption for the radio frequency (RF) chain, $M_{\mathrm{RF}}$ denoting the number of RF chains, and $P_{\text {baseband }}$ denoting the power consumption of the baseband. Herein, we adopt the values from the study [56] as follows: $P_{t}=1 \mathrm{~W}, P_{\mathrm{RF}}=300 \mathrm{~mW}$, and $P_{\text {baseband }}=$ $200 \mathrm{~mW}$. In Figure 12, we show energy efficiency versus SNR. We assume eight users. From the figure, we noticed that the proposed scheme can achieve higher energy efficiency compared to the methods in
$[10,22]$. More importantly, the proposed scheme further reduced the power consumption since it required a single RF chain [57].

(f) Capacity discussion: Figure 13 depicts the cumulative density function (CDF) curves with two distinct SNR values: $5 \mathrm{~dB}$ and $20 \mathrm{~dB}$, with different receive antennas $N_{r}$ and fixed transmit antennas $N_{t}$. We notice that as we increase the SNR values, the CDF curves shift to the right side. This implies that when the SINR is improved, the capacity of the system will be enhanced greatly.

In Table 1, we have tabulated the comparisons between the proposed BM-FDRA scheme and the similar representation beamspace schemes investigated in $[10,22]$.

\section{Conclusion}

This paper investigated a different method to achieve multiuser communications in mmWave networks using the Butler matrix (BM) frequency diverse retrodirective array (BM-FDRA). The proposed BM-FDRA can receive 
and retransmit information at distinct frequencies towards the users. Furthermore, focused angle-range beamforming with the orthogonal property (i.e., no mutual interferences) can be generated by the proposed BM-FDRA to serve multiple users concurrently. The proposed scheme was theoretically evaluated by the following performance metrics, namely, secrecy sum rate, bit error rate (BER), energy efficiency, and system capacity, from the user's perspective. We verify the achievements of the proposed BM-FDRA over the phased-array scheme and methods in $[10,22]$, respectively, via simulation results. Herein, we realize that the fundamental limitation of the proposed BM-FDRA scheme is that the number of desired users that can be supported by the system cannot be larger than $M \times M$. One possible solution as a future work is to integrate the nonorthogonal multiple-access (NOMA) concept. Thus, the number of users that can be supported by the proposed BM-FDRA scheme can be larger than $M \times M$.

\section{Data Availability}

No data were used to support this study.

\section{Conflicts of Interest}

The authors declare that there are no conflicts of interest regarding the publication of this paper.

\section{References}

[1] S. Kutty and D. Sen, "Beamforming for millimeter wave communications: an inclusive survey," IEEE Communications Surveys and Tutorials, vol. 18, no. 2, pp. 949-973, 2015.

[2] Y. Niu, Y. Li, D. Jin, L. Su, and A. V. Vasilakos, "A survey of millimeter wave (mm-wave) communications for 5G: opportunities and challenges," 2015, https://arxiv.org/abs/1502.07228.

[3] J. G. Andrews, S. Buzzi, W. Choi et al., "What will 5G be?" IEEE Journal on Selected Areas in Communications, vol. 32, no. 6, pp. 1065-1082, 2014.

[4] R. Ford, M. Zhang, M. Mezzavilla, S. Dutta, S. Rangan, and M. Zorzi, "Achieving ultra-low latency in $5 \mathrm{G}$ millimeter wave cellular networks," IEEE Communications Magazine, vol. 55, no. 3, pp. 196-203, 2017.

[5] W. Roh, J.-Y. Seol, J. Park et al., "Millimeter-wave beamforming as an enabling technology for $5 \mathrm{G}$ cellular communications: theoretical feasibility and prototype results," IEEE Communications Magazine, vol. 52, no. 2, pp. 106-113, 2014.

[6] J. Zhang, X. Huang, V. Dyadyuk, and Y. Guo, "Massive hybrid antenna array for millimeter-wave cellular communications," IEEE Wireless Communications, vol. 22, no. 1, pp. 79-87, 2015.

[7] M. Xiao, S. Mumtaz, Y. Huang et al., "Millimeter wave communications for future mobile networks," IEEE Journal on Selected Areas in Communications, vol. 35, no. 9, pp. 1909-1935, 2017.

[8] X. Ge, R. Zi, X. Xiong, Q. Li, and L. Wang, "Millimeter wave communications with OAM-SM scheme for future mobile networks," IEEE Journal on Selected Areas in Communications, vol. 35, no. 9, pp. 2163-2177, 2017.

[9] L. Kong, L. Ye, F. Wu, M. Tao, G. Chen, and A. V. Vasilakos, "Autonomous relay for millimeter-wave wireless communications," IEEE Journal on Selected Areas in Communications, vol. 35, no. 9, pp. 2127-2136, 2017.

[10] Q. Xue, X. Fang, M. Xiao, and L. Yan, "Multi-user millimeter wave communications with nonorthogonal beams," IEEE Transactions on Vehicular Technology, vol. 66, no. 7, pp. 5675-5688, 2017.

[11] L. L. Wei, R. Q. Hu, Y. Qian, and G. Wu, "Key elements to enable millimeter wave communications for $5 \mathrm{G}$ wireless systems," IEEE Wireless Communications, vol. 21, no. 6, pp. 136-143, 2014.

[12] C. Perfecto, J. Del Ser, and M. Bennis, "Millimeter-wave V2V communications: distributed association and beam alignment," IEEE Journal on Selected Areas in Communications, vol. 35, no. 9, pp. 2148-2162, 2017.

[13] Q. Xue, X. Fang, and C.-X. Wang, "Beamspace SU-MIMO for future millimeter wave wireless communications," IEEE Journal on Selected Areas in Communications, vol. 35, no. 7, pp. 1564-1575, 2017.

[14] E. G. Larsson, O. Edfors, F. Tufvesson, and T. L. Marzetta, "Massive MIMO for next generation wireless systems," IEEE Communications Magazine, vol. 52, no. 2, pp. 186-195, 2014.

[15] M. S. Gast, 802.11ac: A Survival Guide, O’Reilly Media, Inc., Sebastopol, CA, USA, 1st edition, 2013.

[16] T. Baykas, C.-S. Sum, Z. Lan et al., "IEEE 802.15.3c: the first IEEE wireless standard for data rates over $1 \mathrm{~Gb} / \mathrm{s}$," IEEE Communications Magazine, vol. 49, no. 7, pp. 114-121, 2011.

[17] S. Chia, M. Gasparroni, and P. Brick, "The next challenge for cellular networks: backhaul," IEEE Microwave Magazine, vol. 10, no. 5, pp. 54-66, 2009.

[18] E. Perahia, C. Cordeiro, M. Park, and L. L. Yang, "IEEE 802 11ad: defining the next generation multi-Gbps Wi-Fi," in Proceedings of the IEEE 7th Consumer Communications and Networking Conference (CCNC), pp. 1-5, Las Vegas, NV, USA, January 2010.

[19] Y. Zeng and R. Zhang, "Millimeter wave MIMO with lens antenna array: a new path division multiplexing paradigm," IEEE Transactions on Communications, vol. 64, no. 4, pp. 1557-1571, 2016.

[20] A. Sayeed and J. Brady, "Beamspace MIMO for high-dimensional multiuser communication at millimeter-wave frequencies," in Proceedingsof the IEEE Global Commun. Conference (GLOBECOM), pp. 3679-3684, Atlanta, GA, USA, December 2013.

[21] P. V. Amadori and C. Masouros, "Low RF-complexity millimeter-wave beamspace-MIMO systems by beam selection," IEEE Transactions on Communications, vol. 63, no. 6, pp. 2212-2223, 2015.

[22] X. Gao, L. Dai, Z. Chen et al., "Near-optimal beam selection for beamspace mmwave massive MIMO systems," IEEE Communications Letters, vol. 20, no. 5, pp. 1054-1057, 2016.

[23] Y. Wang, H.-M. Wang, T.-X. Zheng, and Q. Yin, "Secure transmissions in millimeter wave systems," IEEE Transactions on Communications, vol. 65, no. 5, pp. 2114-2127, 2017.

[24] M. Campo, W. Simon, and R. Baggen, "Steerable antenna array at $24 \mathrm{GHz}$ using butler matrices and MEMS-switches," in Proceedings of the of IEEE International Symposium on Antennas and Propagation, pp. 1-2, Kamp-Lintfort, Chicago, IL, USA, July 2012.

[25] G. Tian, J.-P. Yang, and W. Wu, "A novel compact Butler matrix without phase shifter," IEEE Microwave and Wireless Components Letters, vol. 24, no. 5, pp. 306-308, 2014.

[26] Y. Ding and V. Fusco, "Sidelobe manipulation using Butler matrix for $60 \mathrm{GHz}$ physical layer secure wireless communication," in Proceedings of the 2013 Loughborough Antennas 
and Propagation Conference, pp. 61-65, Loughborough, UK, November 2013.

[27] S. Y. Nusenu, H. Chen, W.-Q. Wang, S. Ji, and O. A. K. OpuniBoachie, "Frequency diverse array using Butler matrix for secure wireless communications," Progress In Electromagnetics Research M, vol. 63, pp. 207-215, 2018.

[28] P. Antonik, M. C. Wicks, H. D. Griffiths, and C. J. Baker, "Frequency diverse array radars," in Proceedings of the IEEE National Radar Conference, pp. 215-217, Verona, NY, USA, April 2006.

[29] W.-Q. Wang, H. C. So, and A. Farina, "An overview on time/ frequency modulated array processing," IEEE Journal of Selected Topics in Signal Processing, vol. 11, no. 2, pp. 228-246, 2017.

[30] K. Chen, S. Yang, Y. Chen, and S.-W. Qu, "Accurate models of time invariant beampatterns for frequency diverse array," IEEE Transactions on Antennas and Propagation, vol. 67, no. 5, pp. 3022-3029, 2019.

[31] Z. Wang, Y. Song, T. Mu, and Z. Ahmad, "A short-range range-angle dependent beampattern synthesis by frequency diverse array," IEEE Access, vol. 6, pp. 22664-22669, 2018.

[32] B. Chen, X. Chen, Y. Huang, and J. Guan, "Transmit beampattern synthesis for FDA radar," IEEE Antennas and Wireless Propagation Letters, vol. 17, no. 1, pp. 98-101, 2018.

[33] S. Y. Nusenu, Z. Wang, and W. Q. Wang, "FDA radar using costas sequence modulated frequency increments," in Proceedings of the 2016 CIE International Conference, on Radar, Guangzhou, China, October 2016.

[34] S. Y. Nusenu, W.-Q. Wang, and A. Basit, "Time-modulated FD-MIMO array for integrated radar and communication systems," IEEE Antennas and Wireless Propagation Letters, vol. 17, no. 6, pp. 1015-1019, 2018.

[35] S. Y. Nusenu and W.-Q. Wang, "Dual-function FDA MIMO radar-communications system employing costas signal waveforms," in Proceedings of the 2018 IEEE Radar Conference, pp. 0033-0038, Oklahoma City, OK, USA, April 2018.

[36] S. Y. Nusenu, S. Huaizong, P. Ye, W. Xuehan, and A. Basit, "Dual-function radar-communications system design via sidelobe manipulation based on FDA Butler matrix," IEEE Antennas and Wireless Propagation Letters, vol. 18, no. 3, pp. 452-456, 2019.

[37] S. Y. Nusenu and A. Basit, "Cognitive transmit subarray FDA design for integrated radar-communication using flexible sidelobe control," in Proceedings of the 2018 IEEE 7th International Conference on Adaptive Science and Technology (ICAST), pp. 1-6, Accra, Ghana, August 2018.

[38] S. Y. Nusenu, W.-Q. Wang, and H. Chen, "Dual-function MIMO radar-communications employing frequency-hopping chirp waveforms," Progress in Electromagnetics Research M, vol. 64, pp. 135-146, 2018.

[39] S. Y. Nusenu, W.-Q. Wang, and J. Xiong, "Time-modulated frequency diverse array for physical-layer security," IET Microwaves, Antennas and Propagation, vol. 15, no. 3, pp. 336-345, 2017.

[40] S. Y. Nusenu, W. Q. Wang, and S. Ji, "Secure directional modulation using frequency diverse array antenna," in Proceedings of the IEEE Radar Conference, pp. 378-382, Seattle, WA, USA, May 2017.

[41] S. Y. Nusenu and W.-Q. Wang, "Range-dependent spatial modulation using frequency diverse array for OFDM wireless communications," IEEE Transactions on Vehicular Technology, vol. 67, no. 11, pp. 10886-10895, 2018.

[42] S. Y. Nusenu and A. Basit, "Frequency diverse array antennas: from their origin to their application in wireless communication systems," Journal of Computer Networks and Communications, vol. 2018, Article ID 5815678, 12 pages, 2018.
[43] Y. Ding, V. Fusco, and J. Zhang, "Frequency diverse array OFDM transmitter for secure wireless communication," Electronics Letters, vol. 51, no. 17, pp. 1374-1376, 2015.

[44] V. Fusco and N. Buchanan, "Developments in retrodirective array technology," IET Microwaves, Antennas \& Propagation, vol. 7, no. 2, pp. 131-140, 2013.

[45] R. Y. Miyamoto and T. Itoh, "Retrodirective arrays for wireless communications," IEEE Microwave Magazine, vol. 3, no. 1, pp. 71-79, 2002.

[46] Y. Ding and V. Fusco, "A synthesis-free directional modulation transmitter using retrodirective array," IEEE Journal of Selected Topics in Signal Processing, vol. 11, no. 2, pp. 428-441, 2017.

[47] Y. Ding, J. Zhang, and V. F. Fusco, "Retrodirective-assisted secure wireless key establishment," IEEE Transactions on Communications, vol. 65, no. 1, pp. 320-334, 2017.

[48] N. B. Buchanan, V. F. Fusco, and M. Van Der Vorst, "SATCOM retrodirective array," IEEE Transactions on Microwave Theory and Techniques, vol. 64, no. 5, pp. 1614-1621, 2016.

[49] N. B. Buchanan, V. Fusco, and M. Van Der Vorst, "Phase conjugating circuit with frequency offset beam positioning error correction facility for precision retrodirective antenna applications," in Proceedings of the 41st European Microwave Conference, pp. 1281-1283, Manchester, UK, October 2011.

[50] A.-M. Yao, W. Wu, and D.-G. Fang, "Frequency diverse array phase conjugating retrodirective array with simultaneous range-focusing capability for multi-targets," in Proceedings of the Asia-Pacific Microwave Conference, pp. 1-3, Nanjing, China, December 2015.

[51] W.-Q. Wang, "Retrodirective frequency diverse array focusing for wireless information and power transfer," IEEE Journal on Selected Areas in Communications, vol. 37, no. 1, pp. 61-73, 2019.

[52] R. J. Mailloux, Phased Array Antenna Handbook, Artech House, Inc., Boston, MA, USA, 2005.

[53] F. Oggier and B. Hassibi, "The secrecy capacity of the MIMO wiretap channel," IEEE Transactions on Information Theory, vol. 57, no. 8, pp. 4961-4972, 2011.

[54] J. R. Barry, E. A. Lee, and D. G. Messerschmitt, Digital Communnication, Springer, Berlin, Germany, 3rd edition, 2004.

[55] M. P. Daly and J. T. Bernhard, "Directional modulation technique for phased arrays," IEEE Transactions on Antennas and Propagation, vol. 57, no. 9, pp. 2633-2640, 2009.

[56] X. Gao, L. Dai, S. Han et al., "Energy-efficient hybrid analog and digital precoding for mmWave MIMO systems with large antenna arrays," IEEE Journal on Selected Areas in Communications, vol. 34, no. 4, pp. 998-1009, 2016.

[57] Y. I, V. Fusco, A. Shitvov, Y. Xiao, and H. Li, "Beam index modulation wireless communication with analog beamforming," IEEE Transactions on Vehicular Technology, vol. 67, no. 7, pp. 6340-6354, 2018. 